\title{
Iterative Frequency Domain Equalization and Carrier Synchronization for Multi-Resolution Constellations
}

\author{
P. Pedrosa, R. Dinis, and F. Nunes
}

\begin{abstract}
Broadband broadcast and multicast wireless systems usually employ OFDM modulations (Orthogonal Frequency Division Multiplexing) combined with non-uniform hierarchical constellations. However, these schemes are very prone to nonlinear distortion effects and have high carrier synchronization requirements. SC-FDE (Single-Carrier with Frequency-Domain Equalization) is an attractive alternative for OFDM, especially when an efficient power amplification is intended. In this paper we consider the use of SC-FDE schemes combined with non-uniform hierarchical constellations in broadband broadcast and multicast wireless systems. We study the impact of residual CFO (Carrier Frequency Offset) on the performance of multi-resolution schemes and we propose iterative frequency domain receivers with joint detection and carrier synchronization to cope with residual CFO estimation errors (a coarse CFO estimation and compensation is assumed before the equalization procedure). Our results show that while a very high carrier synchronization accuracy is required for the least protected bits, the most protected bits are relatively robust to the CFO. By employing the proposed receiver we increase significantly the robustness to residual CFO estimation errors.
\end{abstract}

Index Terms-Carrier frequency offset, multi-resolution transmission, non-uniform hierarchical QAM, single carrier with frequency-domain equalization.

\section{INTRODUCTION}

$\mathbf{I}$ $\mathrm{N}$ BROADCAST and multicast wireless systems we transmit the same information to several users which can have substantially different propagation conditions. It can be shown [1] that it is possible to exchange some of the capacity of the good communication links to the poor ones. A very simple method to improve the efficiency of the network is to use non-uniform hierarchical constellations which are able to provide bit streams with different error protections [2]. This means that we have two or more classes of bits with different error protections. A given user can attempt to demodulate only the more protected bits or also the other bits that carry the additional information, depending on the channel conditions; we can also have low-cost terminals only able to receive the most protected bits. An application of these techniques is in the transmission of coded voice or video signals, where different

Manuscript received June 10, 2008; revised August 19, 2010; accepted August 19, 2010. Date of publication September 23, 2010; date of current version November 19, 2010. This work was supported in part by the FCT-Fundação para a Ciência e Tecnologia under pluriannual funding, by the ADCOD project PTDC/EEA - TEL/099973/2008, by the U-BOAT project PTDC/EEA TEL/67066/2006, and by the Ph.D. Grant SFRH / BD / 40265 / 2007.

P. Pedrosa and F. Nunes are with the Instituto de Telecomunicações, and also with the Instituto Superior Técnico, Universidade Técnica de Lisboa, 1040-001 Lisboa, Portugal (e-mail: ppedrosa@1x.it.pt; nunes@1x.it.pt).

R. Dinis is with the Instituto de Telecomunicações, 1040-001 Lisboa, Portugal and also with the Faculdade de Ciências e Tecnologia, Universidade Nova de Lisboa, 2829-516 Caparica, Portugal (e-mail: rdinis@1x.it.pt).

Color versions of one or more of the figures in this paper are available online at http://ieeexplore.ieee.org.

Digital Object Identifier 10.1109/TBC.2010.2073251 error protections may be associated to different resolutions [2], [3].

An important drawback of non-uniform signal constellations is that they are very sensitive to residual interference. This can be the residual ISI (Inter-Symbol Interference) at the output of a practical equalizer that does not invert completely the channel effects (e.g., an equalizer optimized under the MMSE (Minimum Squared Mean Error) [4]). Since phase errors lead to significant performance degradation, these constellations are also very sensitive to carrier frequency errors.

For broadband broadcast and multicast wireless systems the time-dispersion effects associated to the multipath propagation can be severe. In this case, conventional time-domain equalization schemes are not practical. Alternative techniques employing block transmission with appropriate cyclic extensions and employing FDE (Frequency-Domain Equalization) techniques have been shown to be suitable for high data rate transmission over severely time-dispersive channels without requiring complex receivers. For this reason, OFDM (Orthogonal Frequency Division Multiplexing) modulations [5] are usually selected for these systems [6], [7]. However, the OFDM signals have high envelope fluctuations and a high PMEPR (Peak-to-Mean Envelope Power Ratio) leading to amplification difficulties. For this reason, several techniques have been proposed for reducing the envelope fluctuations of OFDM signals (see [8] and references within), although the transmitted signals still have higher envelope fluctuations than SC (Single-Carrier) signals based on similar constellations. Moreover, since the subcarrier spacing is a very small fraction of the transmission bandwidth, the carrier synchronization requirements are also much higher for OFDM modulations.

An alternative approach based on the same block transmission principle is SC modulations (Single-Carrier) combined with FDE (also denoted SC-FDE) [9]. When compared with OFDM, SC-FDE has the advantage of reduced envelope fluctuations and higher robustness to carrier frequency errors (contrarily to OFDM schemes, where frequency errors lead to ICI [10]-[12], for SC-FDE the CFO induces a rotation in the constellation that grows linearly along the block). The performance of SC-FDE can be further improved if the traditional linear FDE is replaced by an IB-DFE (Iterative Block-Decision Feedback Equalizer) [13]. An IB-DFE receiver with joint post-equalization carrier frequency synchronization was proposed in [14], which can be regarded as a modified turbo equalization scheme where, for each iteration, we perform DD (Decision-directed) CFO (Carrier Frequency Offset) estimation, allowing high robustness against residual CFO.

Earlier IB-DFE implementations considered hard-decisions (weighted by the blockwise reliability) in the feedback loop. To improve the performance and allow truly turbo FDE implementations, IB-DFE schemes with soft decisions were proposed 
[15]-[17] usually only for QPSK constellations. The extension for larger constellations leads to difficulties on the computation of the reliability of each block, as well as problems on the computation of the average symbol values conditioned to the FDE and the channel decoder output. Notably, carrier synchronization for QAM differs from carrier synchronization for QPSK specially for large constellations which are particularly sensitive to CFO. In [18] SC-FDE schemes with IB-DFE receivers were considered and a general method for the computation of the receiver parameters for any constellation was proposed. The approach in [18] relies on an analytical characterization of the mapping rule where the constellation symbols are written as a linear function of the transmitted bits. This method is then employed in both uniform and non-uniform hierarchical QAM constellations.

There are two major contributions of this paper. First, we consider the use of SC-FDE schemes combined with multiresolution for broadband broadcast and multicast wireless systems, in opposition to most works on multi-resolution that consider OFDM implementations. Second, we study the impact of residual CFO (Carrier Frequency Offset) on the performance of multi-resolution schemes. We assume that a coarse CFO estimation and compensation is made before the equalization procedure, but the coarse CFO estimate can have a significant error leading to a residual CFO. To cope with this residual CFO, we present and evaluate an iterative frequency domain receiver with joint detection and carrier synchronization.

This paper is organized in following manner: Section II pertains to the description of the system, with subsections dedicated to the mapping rules, the receiver scheme and the computation of the parameters of the receiver; Section III reports the performance results; and finally Section IV concludes this paper.

\section{SYSTEM DESCRIPTION}

\section{A. Mapping Rules}

Assume the transmitted symbols $s_{n}$ belong to a given alphabet $\mathcal{G}$ (i.e. a given constellation) with dimension $M=\# \mathcal{G}$ and are selected according to the corresponding bits $\beta_{n}^{(m)}, m=$ $1,2, \ldots, \mu\left(\mu=\log _{2}(M)\right)$. i.e., $s_{n}=f\left(b_{n}^{(1)}, b_{n}^{(2)}, \ldots, b_{n}^{(\mu)}\right)$, with $b_{n}^{(m)}=2 \beta_{n}^{(m)}-1$. We assume that $\beta_{n}^{(m)}$ is the $m$ th bit associated to the $n$th symbol and $b_{n}^{(m)}$ is the corresponding polar representation, i.e., $\beta_{n}^{(m)}=0$ or 1 and $b_{n}^{(m)}=-1$ or +1 , respectively.

For 4-PAM constellations and Gray mapping it can be shown that $[18]$

$$
s_{n}=d_{1} b_{n}^{(2)}+d_{2} b_{n}^{(1)} b_{n}^{(2)},
$$

If $k=d_{2} / d_{1}=0.5$ we have that (1) is uniform whereas if $0<k<0.5$ then (1) is non-uniform and truly hierarchical [2]. For 8-PAM constellations and Gray mapping we have

$$
s_{n}=d_{1} b_{n}^{(3)}+d_{2} b_{n}^{(3)} b_{n}^{(2)}+d_{3} b_{n}^{(3)} b_{n}^{(2)} b_{n}^{(1)} .
$$

Again, if $k=d_{2} / d_{1}=d_{3} / d_{2}=0.5$ (2) is uniform ${ }^{1}$ whereas if $0<k<0.5$ (2) is non-uniform and truly hierarchical. Clearly, our PAM constellations can be written as the sum of several

\footnotetext{
${ }^{1}$ Actually we could have two different ratios $k_{1}=d_{2} / d_{1}$ and $k_{2}=d_{3} / d_{2}$.
}

binary polar constellations with amplitudes $d_{1}, d_{2}$, etc, and $k$ is a parameter that characterizes the "non-uniform" degree of the constellations.

It should be mentioned that hierarchical QAM constellations have higher PMEPR than QPSK constellations. However, their PMEPR is lower than for uniform QAM and for systems with very different error protections $(k \ll 0.5)$ the PMEPR can be close to the one with QPSK. Moreover, the PMEPR is much lower for SC-FDE than for OFDM with the same constellation (hierarchical or not).

If the transmitted symbols are selected from a QAM constellation under a Gray mapping rule the $M$-QAM constellation is written as the sum of two PAM constellations each with dimension $\sqrt{M}$, one for the in-phase (real) component and the other for the quadrature (imaginary) component. Therefore, the corresponding mapping is straightforward: half of the bits are used to define the in-phase component and the other half is used to define the quadrature component. Considering a square constellation we obtain for 16-QAM

$$
s_{n}=d_{1} b_{n}^{(2)}+d_{2} b_{n}^{(1)} b_{n}^{(2)}+j\left(d_{1} b_{n}^{(4)}+d_{2} b_{n}^{(3)} b_{n}^{(4)}\right),
$$

and for 64-QAM

$$
\begin{aligned}
s_{n}=d_{1} b_{n}^{(3)}+ & d_{2} b_{n}^{(3)} b_{n}^{(2)}+d_{3} b_{n}^{(3)} b_{n}^{(2)} b_{n}^{(1)} \\
& +j\left(d_{1} b_{n}^{(6)}+d_{2} b_{n}^{(6)} b_{n}^{(5)}+d_{3} b_{n}^{(6)} b_{n}^{(5)} b_{n}^{(4)}\right)
\end{aligned}
$$

\section{B. IB-DFE Receiver With Joint Carrier Synchronization}

Considering Fig. 1, the received time domain block, $\left\{y_{n}^{\prime} ; n=\right.$ $0,1, \ldots, N-1\}$, is passed to the frequency domain by a DFT operation, leading to the block $\left\{Y_{k}^{\prime} ; k=0,1, \ldots, N-1\right\}$, with

$$
Y_{k}^{\prime}=S_{k}^{\prime} H_{k}+N_{k},
$$

where $H_{k}$ and $N_{k}$ denote the channel transfer function and the channel noise, respectively, for the $k$ th subchannel. The block of frequency-domain symbols $\left\{S_{k}^{\prime} ; k=0,1, \ldots, N-1\right\}$ is the DFT of the transmitted time-domain block, $\left\{s_{n}^{\prime} ; n=0,1, \ldots, N-1\right\}$, with

$$
s_{n}^{\prime}=s_{n} \exp \left(j 2 \pi \frac{\Delta f n T}{N}\right)
$$

where $s_{n}$ denotes the $n$th data symbol to be transmitted, selected from a given constellation (e.g. a QAM or a PSK constellation), $T$ is the duration of the block, and $\Delta f$ is the CFO.

For a given iteration $i$, the frequency-domain samples at the output of the FDE are given by

$$
\tilde{S}_{k}^{(i)}=F_{k}^{(i)} Y_{k}^{\prime}-B_{k}^{(i)} \bar{S}_{k}^{(i-1)},
$$

where $\left\{F_{k}^{(i)} ; k=0,1, \ldots, N-1\right\}$ are the feedforward coefficients and $\left\{B_{k}^{(i)} ; k=0,1, \ldots, N-1\right\}$ are the feedback coefficients. $\left\{\bar{S}_{k}^{(i-1)} ; k=0,1, \ldots, N-1\right\}$ denotes the DFT of the block of the time-domain average symbol values associated to the previous iteration, $\left\{\bar{s}_{n}^{(i-1)} ; n=0,1, \ldots, N-1\right\}$.

The DD estimation of the carrier frequency offset can be implemented using

$$
\widehat{\Delta f}=\frac{N}{2 \pi M T} \arg \{\xi\}
$$

